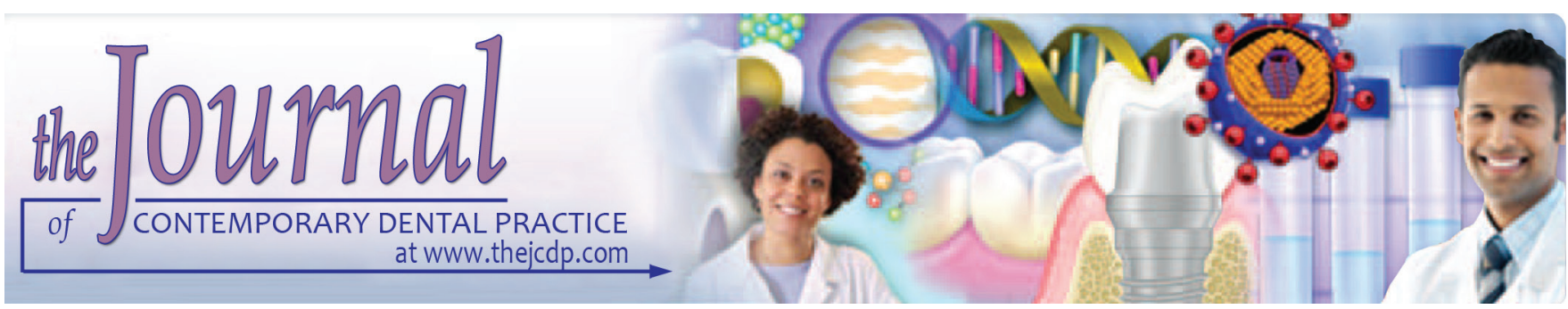

\title{
Lingual Frenectomy in Joubert Syndrome
}

\author{
${ }^{1}$ Vivek Gurjar, ${ }^{2}$ Minal Gurjar, ${ }^{3}$ Channaveer Pattanshetti, ${ }^{4}$ Banashree Sankeshwari
}

\section{ABSTRACT}

Aim: Our aim is to present a case of a rare disorder, such as Joubert syndrome (JS) which required oral surgical intervention under general anesthesia at a very young age to help in achieving normal developmental milestones.

Background: Ciliopathies are an emerging class of diseases of which JS is a significant member. There have been very few cases of JS with its distinguishing features which have been reported in recent literature.

Case report: We herewith present a case of JS who reported to our unit with a complaint of speech abnormality. Intraoral examination revealed a tongue-tie which was planned for surgical release. As any drugs that interfere with respiratory depression have to be used with utmost care, adequate precautions were taken in this case and the patient was treated under general anesthesia. The tongue-tie was released and associated hamartomatous outgrowths from the floor of the mouth were also excised. Appropriate postoperative care was taken and the patient was discharged.

Conclusion: The patient treated by us did not reveal any significant intraoperative or postoperative complications. Treatment of cases of JS required a team effort of multiple dental and medical specialists. Long-term follow-up of such patients is required so that they achieve normal chronological development.

Clinical significance: Joubert syndrome being a rare disorder requires special anesthetic care during any surgical procedure, especially that involving the oral cavity. The need and timing of

\footnotetext{
${ }^{1,3}$ Department of Oral and Maxillofacial Surgery, Bharati Vidyapeeth Deemed University Medical College and Hospital Sangli, Maharashtra, India

${ }^{2}$ Department of Periodontics, Bharati Vidyapeeth Deemed University Medical College and Hospital, Sangli, Maharashtra India

${ }^{4}$ Department of Prosthodontics, Bharati Vidyapeeth Deemed University Medical College and Hospital, Sangli, Maharashtra India

Corresponding Author: Vivek Gurjar, 233 E, Surve Colony Tarabai Park, Kolhapur, Maharashtra, India, e-mail: vivek_ gurjar@yahoo.com
}

surgical intervention in such cases should be carefully assessed and appropriate precautions should be taken.

Keywords: Frenectomy, Joubert syndrome, Polydactyly, Tongue-tie.

How to cite this article: Gurjar V, Gurjar M, Pattanshetti C, Sankeshwari B. Lingual Frenectomy in Joubert Syndrome. J Contemp Dent Pract 2017;18(8):728-731.

\section{Source of support: Nil}

\section{Conflict of interest: None}

\section{INTRODUCTION}

Joubert syndrome (JS) is a condition that manifests in various organs in the patient. Clinical features in this disorder may vary significantly in individuals affected, even though it occurs in the same family. Other names for this condition are cerebello-oculo-renal syndrome, JoubertBoltshauser syndrome, and familial aplasia of the vermis.

A typical feature of JS, i.e., seen on cerebral imaging investigations, such as magnetic resonance imaging (MRI) is a brain abnormality called the molar tooth sign. This sign is named so because the brain abnormality resembles a cross section of a molar dentition when seen on an MRI. ${ }^{1}$ Distinct facial features that characterize JS include a triangular-shaped mouth, a broad forehead, high arched eyebrows, widely spaced eyes, ptosis, and lowly set ears, which were all seen in the case presented. ${ }^{2}$

The most common presenting feature in the newborn period of affected infants is an abnormal breathing pattern characterized as episodic hyperpnea. This characteristic feature was also reported in our case. A systematic headto-toe physical examination is essential to identify such patients.

In JS, the cerebellar vermis and brain stem of the brain are not completely developed during pregnancy because of a faulty gene. The cerebellar vermis controls posture, muscle tone, and head and eye movements. The brain stem is responsible for functions, such as heartbeats, breathing, and temperature regulation. Thus, 
underdevelopment of the cerebellar vermis and brain stem invariably affects their normal function and shows the manifestations that are typical of JS.

There is no treatment, i.e., specific for JS. Affected individuals require supportive care for developmental disabilities, such as physical and occupational therapies. Speech therapy should be given where cognitive development is undermined. Management for seizures with appropriate drugs is given where it is required.

\section{CASE REPORT}

A 4-year-old female patient reported to our unit with a chief complaint of multiple irregularly arranged carious teeth in both the upper and lower jaws (Fig. 1). The patient also complained of difficulty in movement of the tongue.

The patient was an offspring of a result of a consanguineous marriage with a history of full-term cesarean delivery. She had an elder sibling with no relevant medical history. The parents also reported no significant medical history. The patient's parents gave a history of delayed developmental milestones of the child.

On examination, the patient had an average stature, weighed $12.4 \mathrm{~kg}$, and had an abnormal gait. The head appeared to be slightly brachycephalic with frontal prominence and prominent metopic sutures. The face

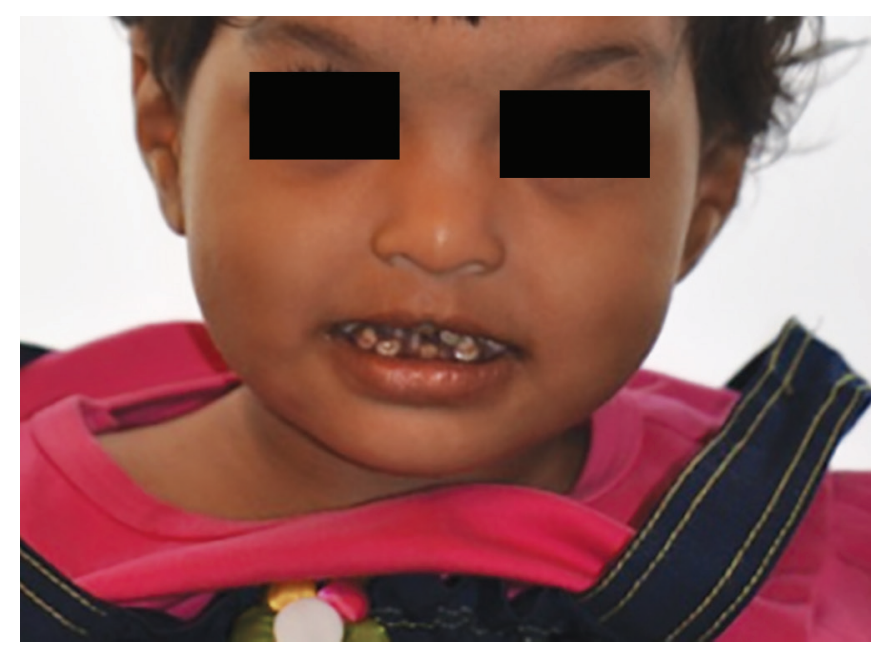

Fig. 1: Patient showing features of JS

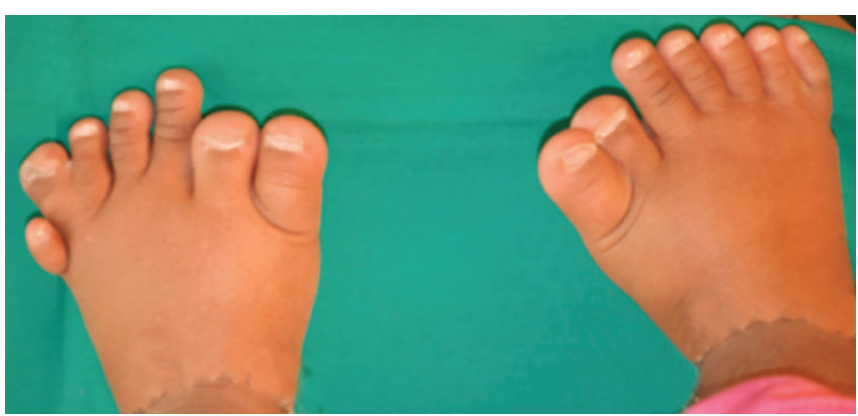

Fig. 3: Polydactyl feet appeared to be asymmetric in appearance with midface flattening. The eyes showed mild hypertelorism. Speech was abnormal due to obstruction of tongue movements as a result of the tongue-tie that was observed (Fig. 2).

Intraoral examination revealed a high-arched grooved palate. The tongue was lobulated with multiple hamartomatous growths in addition to the presence of a tonguetie. Multiple supernumerary teeth were also noted in the lower anterior and upper and lower posterior regions of the jaw.

The extremities showed a polydactyl nature (Figs 3 and 4).

After routine preoperative investigations, the patient was posted for a planned surgical release of the tonguetie under general anesthesia along with rehabilitative dental treatment (Fig. 5). Excision of the hamartomatous outgrowths from the floor of the mouth was also carried out (Fig. 6). The patient was followed up for 1 month thereafter.

\section{DISCUSSION}

From a clinical point of view, there is limited knowledge regarding the natural history of JS. This syndrome is very rare and was reported initially rather recently. It was named after the French neurologist Marie Joubert

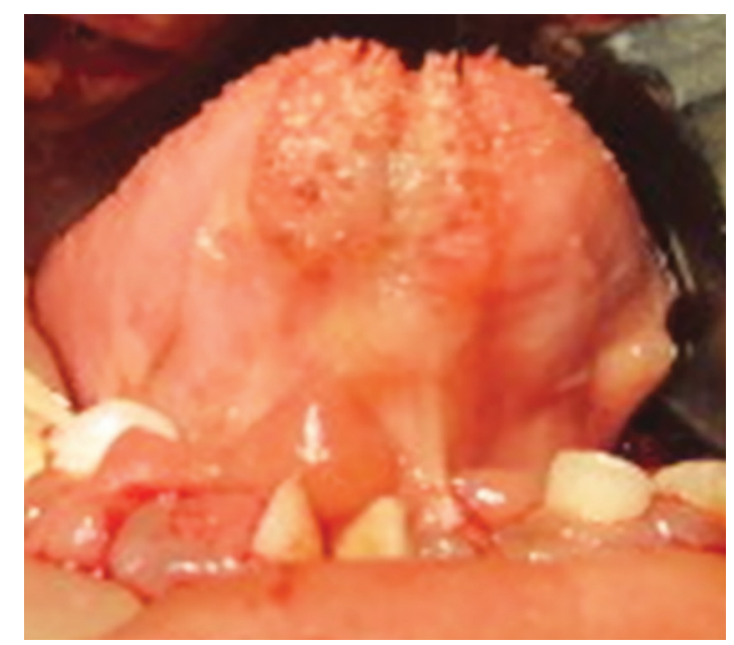

Fig. 2: Tongue-tie

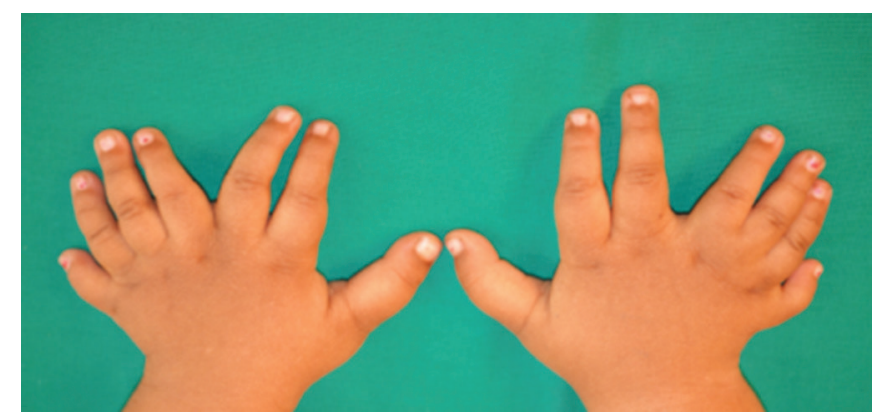

Fig. 4: Polydactyl hands 


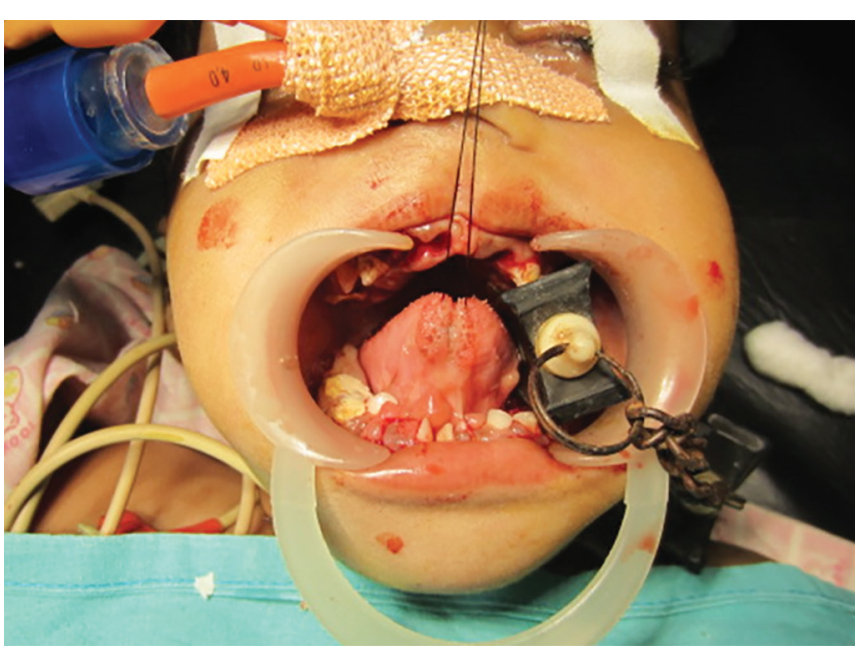

Fig. 5: Intraoperative view

who first described it in 1969. Individuals affected by this syndrome usually present in early childhood, ataxia, dysmorphic facies, global developmental delay, hypotonia, tachypnea, and oculomotor apraxia. ${ }^{3}$ There has also been observed to be an association between renal and retinal findings, although not in every case. Episodes of tachypnea and bradypnea during infancy and abnormal ocular movements also have been noted in a few cases. ${ }^{4-6}$ The striking hyperpnea in infants with JS sets it apart from other similar conditions. Individuals affected show mild-to-severe delayed mental development and intellectual disability with a significant range in intelligence quotient between 30 and 80 . However, the presence of speech and motor deficits makes this assessment quite difficult. ${ }^{7}$ Most children achieve standard milestones in development though usually at a much later age. When behavioral disturbances are present, they usually reflect as impulsive reactions and uncontrollable tempers. ${ }^{8}$

Skeletal changes seen in this syndrome reflect as polydactyly that may be seen in up to $16 \%$ of cases reported. This feature was also observed in our case. Postaxial polydactyly and preaxial polydactyly in toes were commonly noted. In the presence of signs of oral-facial-digital syndrome type VI, mesoaxial polydactyly has been almost always observed. ${ }^{9}$

Joubert syndrome is an autosomal recessive inherited condition. The affected individual has mutations in both copies of her genes. Parents of the individual affected are both carriers because they have one nonworking copy and a working copy of the JS gene. Therefore, there is a $25 \%$ probability with each pregnancy that the progeny will be affected by JS. Around 10 genes have been implicated to a number of disorders related to JS known as ciliopathies. ${ }^{10}$ Alterations in these genes are not always the only cause in a few cases with JS, indicating that some other genetic causes may still be unidentified. An area of the rhombic lips located in the superior-lateral

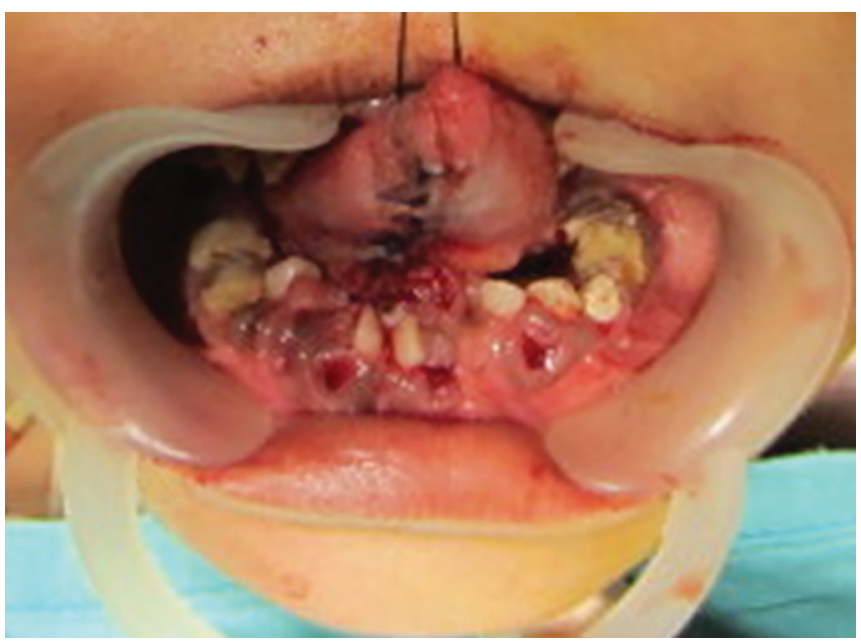

Fig. 6: Postoperative view

area of the brain stem gives rise to cells that migrate to form various neurons of the brain including the inferior olives, neurons of the basis pontis, and arcuate nuclei. These cells displace each other as they migrate to their specific permanent positions. It is postulated that an abnormality in this process may be related to JS as these structures are noted to be abnormal.

It is usually recommended to have annual pediatric ophthalmic and neurological evaluation along with monitoring of growth, sexual maturation, and endocrinological evaluation.

Individuals affected with progressive kidney failure should also undergo investigations, such as complete blood count, urinalysis, blood urea nitrogen, creatinine level assessment, abdominal ultrasound, and blood pressure measurements yearly till the age of 20 . As the disease progresses, findings on an ultrasound show echogenic and scarred renal changes. These two changes in the kidney are considered to be distinctive features of JS. However, a literature review showed that cystic dysplasia in the kidneys in individuals with Dekaban-Arima syndrome was found to be indistinguishable as compared with the features of nephronophthisis. ${ }^{11}$

Joubert syndrome does not have any specific outline of treatment. Patient care is mainly supportive, and regular intervention for cases with developmental disabilities includes physiotherapy, occupational and speech therapy, and management for epilepsy where required.

There are not much data available on the long-term outcome of individuals with JS. Persistent neuromotor developmental retardation is seen most commonly in surviving patients.

Postbirth, prognosis of infants with JS is dependent on the amount and extent of respiratory changes. Repeated incidences of long periods of apnea could be a threat to life requiring ventilator support. In many instances, respiratory abnormalities tend to spontaneously resolve in the 
first year of life itself. Prognosis thereafter depends on complications seen in the liver and kidney which, if not identified and treated early, represents the main reason of fatality in children with JS.

\section{CONCLUSION}

A major challenge in cases of JS is diagnosing the causative genes and using the findings of research to translate into treatment guidelines for affected individuals and support for family members. At present, there is no known cure for JS. Individuals with JS should see the appropriate medical or dental specialist necessary to help monitor their various clinical issues, namely, a nephrologist, geneticist, ophthalmologist, neurologist, or any other primary care provider. One should be careful while administering any agents that can cause respiratory depression since patients with JS are quite sensitive to their effects. ${ }^{12}$

\section{REFERENCES}

1. Brancati F, Dallapiccola B, Valente EM. Joubert syndrome and related disorders. Orphanet J Rare Dis 2010 Jul;5:20.

2. Maria BL, Boltshauser E, Palmer SC, Tran TX. Clinical features and revised diagnostic criteria in Joubert syndrome. J Child Neurol 1999 Sep;14(9):583-590.

3. Steinlin M, Blaser S, Boltshauser E. Cerebellar involvement in metabolic disorders: a pattern-recognition approach. Neuroradiology 1998 Jun;40(6):347-354.
4. Saraiva JM, Baraitser M. Joubert syndrome: a review. Am J Med Genet 1992 Jul;43(4):726-731.

5. Steinlin M, Schmid M, Landau K, Boltshauser E. Follow-up in children with Joubert syndrome. Neuropediatrics 1997 Aug;28(4):204-211.

6. Joubert M, Eisenring JJ, Robb JP, Andermann F. Familial agenesis of the cerebellar vermis. A syndrome of episodic hyperpnea, abnormal eye movements, ataxia, and retardation. Neurology 1969 Sep;19(9):813-825.

7. Doherty D. Joubert syndrome: insights into brain development, cilium biology, and complex disease. Semin Pediatr Neurol 2009 Sep;16(3):143-154.

8. Hodgkins PR, Harris CM,ShawkatFS, Thompson DA, ChongK, Timms C, Russell-Eggitt I, Taylor DS, Kriss A. Joubert syndrome: long-term follow-up. Dev Med Child Neurol 2004 Oct;46(10):694-699.

9. Gleeson JG, Keeler LC, Parisi MA, Marsh SE, Chance PF, Glass IA, Graham Jr JM, Maria BL, Barkovich AJ, Dobyns WB. Molar tooth sign of the midbrain-hindbrain junction: occurrence in multiple distinct syndromes. Am J Med Genet A 2004 Mar;125A(2):125-134.

10. Poretti A, Huisman TA, Scheer I, Boltshauser E. Joubert syndrome and related disorders: spectrum of neuroimaging findings in 75 patients. AJNR Am J Neuroradiol 2011 Sep;32(8):1459-1463.

11. Kumada S, Hayashi M, Arima K, Nakayama H, Sugai K, Sasaki M, Kurata K, Nagata M. Renal disease in Arima syndrome is nephronophthisis as in other Joubert-related cerebello-oculo-renal syndromes. Am J Med Genet A 2004 Nov;131(1):71-76.

12. van Beek EJ, Majoie CB. Case 25: Joubert syndrome. Radiology 2000 Aug;216(2):379-382. 